\title{
Oral candidiasis and denture stomatitis in diabetic patients: Systematic review and meta-analysis
}

\author{
Loyse MARTORANO-FERNANDES(a) (iD \\ Louise Morais DORNELAS-FIGUEIRA ${ }^{(a)}$ (iD \\ Raissa Micaella MARCELLO-MACHADO(a) (iD \\ Raíra de Brito SILVA(a) (ID \\ Marcela Baraúna MAGNO(b) (iD \\ Lucianne Cople MAIA ${ }^{(b)}$ (D)
}

Altair Antoninha DEL BEL CURY(a)

(a) Universidade Estadual de Campinas Unicamp, Piracicaba Dental School, Department of Prosthodontics and Periodontology, Piracicaba, SP, Brazil.

(b) Universidade Federal do Rio de Janeiro UFRJ, Department of Pediatric Dentistry and Orthodontics, RJ, Brazil.

Declaration of Interests: The authors certify that they have no commercial or associative interest that represents a conflict of interest in connection with the manuscript.

Corresponding Author: Altair Antoninha Del Bel Cury E-mail: altair@unicamp.br

hitps://doi.org/10.1590/1807-3107bor-2020.vol34.0113

Submitted: February 14, 2020

Accepted for publication: June 23, 2020

Last revision: Juy 14, 2020
Abstract: Here, the prevalence of oral candidiasis and denture stomatitis among diabetic patients compared to healthy ones was summarized through a systematic review with meta-analysis. Medline, Scopus, Web of Science, Lilacs, Cochrane Library, Embase, and the grey literature were searched without restriction, until May 2020. Eligibility criteria were established, data were extracted, and quality assessment was conducted by two trained examiners. Qualitative synthesis was based on the recommendations of Fowkes and Fulton. Two meta-analyses were performed on studies investigating patients with: a) oral candidiasis and b) denture stomatitis. Out of 6034 screened studies, seven were eligible for qualitative and quantitative synthesis; of these, three evaluated oral candidiasis and four evaluated denture stomatitis. Qualitative synthesis showed that the main methodological problems of the studies included sample size, source of controls, matching, and randomization. Diabetic patients had a similar chance of developing oral candidiasis to non-diabetic patients (OR1.40 [0.96; 2.04], $\left.\mathrm{p}=0.08, \mathrm{I}^{2}=94 \%\right)$. However, diabetic patients had a higher chance to present denture stomatitis compared to non-diabetic patients (OR 1.92 $\left.[1.42,2.59] \mathrm{p}<0.0001, \mathrm{I}^{2}=0 \%\right)$. Therefore, diabetic patients have a higher chance of developing denture stomatitis compared to non-diabetic patients. However, for all analyses, the certainty of the evidence was considered to be very low.

Keywords: Diabetes Mellitus; Candidiasis, Oral; Stomatitis, Denture; Oral Health.

\section{Introduction}

Diabetes mellitus (DM) is a metabolic chronic disorder caused by the dysfunction of pancreatic islet $\beta$ cells, ${ }^{1}$ in which glucose plasma levels remain high for a prolonged period. This disease affects more than 425 million people worldwide, with equal rates in both genders. ${ }^{2}$ Out of the two types of diabetes, type 2 (non insulin-dependent diabetes mellitus) affects $90 \%$ of people with DM, and is mainly caused by lifestyle, including high-calorie diets, low physical activity, and smoking. ${ }^{3}$

DM has multifactorial characteristics, and is usually associated with systemic complications, ${ }^{4}$ such as hypertension, ${ }^{5}$ kidney disease, ${ }^{6}$ eye disease, ${ }^{7}$ recurrent fungal skin infection, ${ }^{8}$ and oral diseases, including 
gingivitis, periodontitis, ${ }^{9}$ and oral lesions caused by biofilm. ${ }^{10}$ Biofilm infections result in diabetic individuals having a prevalence around $30 \%$ and $58 \%$ of oral candidiasis and denture stomatitis, respectively. ${ }^{11,12}$ Patients with DM commonly use systemic medication, such as antihypertensive and diuretic drugs, ${ }^{13}$ which might decrease salivary flow and, thus, facilitate the accumulation of biofilm. Under these conditions and in association with poor oral hygiene, biofilm matures, facilitating the establishment of various diseases. ${ }^{10}$

Candida albicans is the most prevalent microorganism in the biofilm of oral candidiasis and denture stomatitis. ${ }^{14}$ Candida albicans is a polymorphic fungus that can penetrate the oral mucosa barrier and invade the bloodstream in its hyphal form. ${ }^{14}$ One in vitro study showed that glucose levels in the blood of physiologically normal humans $(0.1 \%)$ is sufficient to enhance the expression level of hypha-associated genes. ${ }^{15}$ Consequently, higher glucose levels, as found in patients with DM, might induce the hyphae form of C. albicans, facilitating the development of the disease.

Denture stomatitis is one of the clinical forms of the oral candidiasis. Although the predisposing factors are multifactorial and distinct, both conditions have a $C$. albicans biofilm as an etiologic factor. ${ }^{16}$ To investigate the predisposing factors, several studies ${ }^{17,18,19,20}$ have evaluated the relationship between these conditions in diabetic individuals. These studies demonstrated a higher prevalence of oral candidiasis and denture stomatitis in diabetic patients compared to healthy patients..$^{1718,19,20}$ However, the quality of the evidence might be inconsistent. For instance, these studies had participants with confounding factors, such as alcohol consumption, ${ }^{17}$ smoking ${ }^{18,19}$ and other cormobities, ${ }^{20}$ consequently, the prevalence of oral candidiasis and denture stomatitis might have been higher. Therefore, the methodological flaws of these studies meant it was not possible to determine whether the prevalence of oral candidiasis and denture stomatitis are associated in diabetic patients. Yet, such evidence could help guide multiprofessional teams, especially dentists, in preventing oral candidiasis and denture stomatitis in patients with DM. Therefore, this study aimed to summarize scientific evidence, through a systematic review and meta-analysis, on the prevalence of oral candidiasis and denture stomatitis in patients with diabetes mellitus compared to non-diabetic patients.

\section{Methodology}

This systematic review was performed according to the recommendations of the Preferred Reporting Items for Systematic Reviews and Meta-Analysis (PRISMA) guidelines. ${ }^{21,22}$ The protocol was registered in the PROSPERO database under the number CRD42018106504.

\section{Literature search strategy}

To identify the primary studies, a search was conducted independently by two examiners in the following electronic databases: PubMed (MEDLINE), Scopus, Web of Science, Lilacs, Cochrane Library, System for Information on Gray Literature in Europe (SiGLE), and Embase. Articles published up to May 2020 were searched comprehensively, without any restrictions on the year or language of publication. The search strategy was suited to each database. The MeSH terms used for the search were "Diabetes Mellitus", “Diabetes Mellitus, Type I", “Diabetes Mellitus, Type II", "Candidiasis, Oral," and "Stomatitis, Denture". In addition, free terms related to the topic were included, using the Boolean operators "AND" and "OR" to combine search terms (Table 1). To explore the literature as widely as possible, hand-searches were also performed of the list of references in the included articles.

\section{Selection of studies and eligibility criteria}

Based on the eligibility criteria of the Population, Exposure, Comparison, and Outcomes (PECO) acronym, ${ }^{22}$ this systematic review included studies that evaluate the prevalence of oral candidiasis and denture stomatitis in patients with diabetes mellitus compared to non-diabetic patients. After searching the databases, the retrieved studies were imported to Mendeley Desktop software (Elsevier, 1.19/2018 version), where all duplicates were removed. Titles and abstracts retrieved from the databases were screened, and full texts were read after applying the eligibility criteria. In cases where the title and 
Table 1. Search strategy based on the databases assessed in the present study.

\begin{tabular}{|c|c|}
\hline Database & Strategy \\
\hline PubMed & 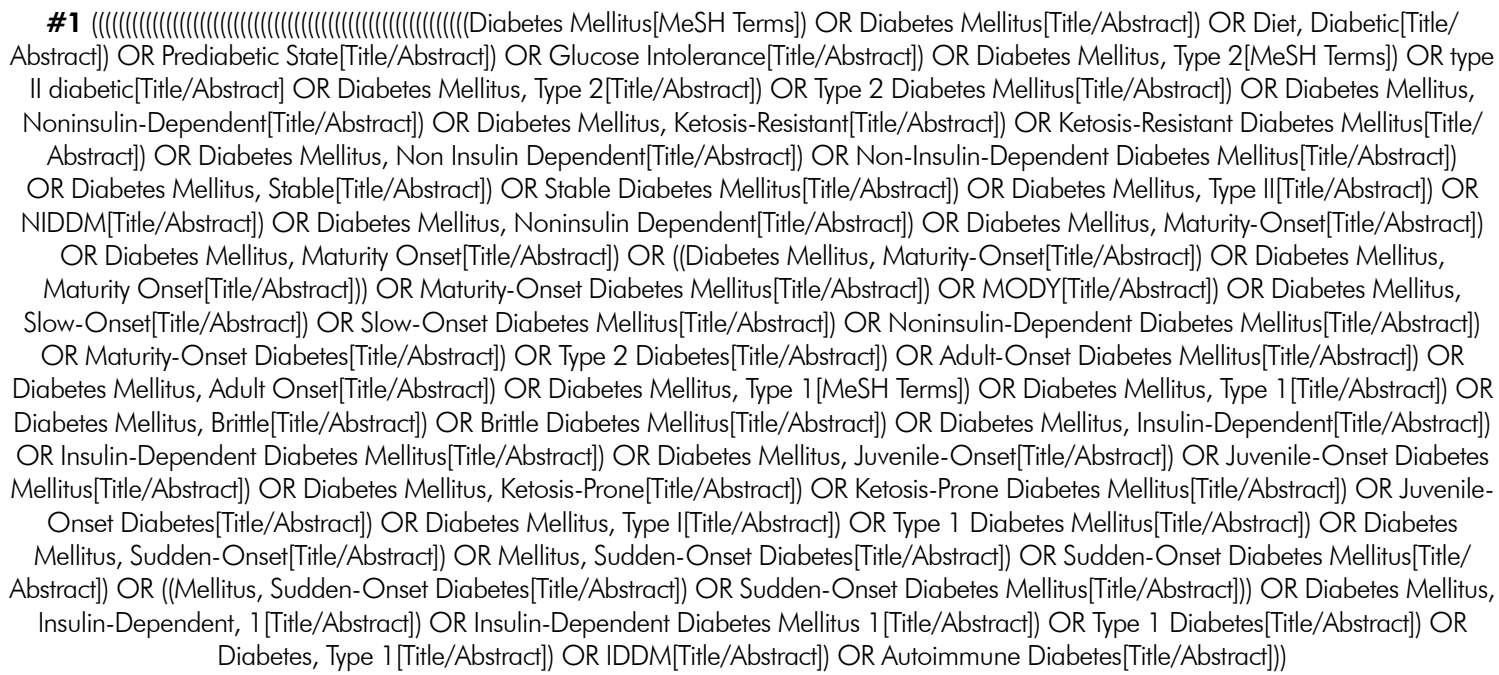 \\
\hline
\end{tabular}

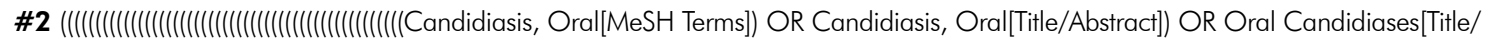
Abstract]) OR Oral Candidiasis[Title/Abstract]) OR Thrush[Title/Abstract]) OR Moniliasis, Oral[Title/Abstract]) OR Moniliases, Oral[Title/ Abstract]) OR Oral Moniliases[Title/Abstract]) OR Oral Moniliasis[Title/Abstract]) OR Candida albicans[MeSH Terms]) OR Candida albicans[Title/Abstract]) OR Candida albican[Title/Abstract]) OR ((Candida albicans[Title/Abstract]) OR Candida albican[Title/Abstract])) OR albicans, Candida[Title/Abstract]) OR Candida glabrata[Title/Abstract]) OR Candida glabrata[MeSH Terms]) OR Candida glabratas[Title/Abstract]) OR ((Candida glabratas[Title/Abstract]) OR glabratas, Candida[Title/Abstract])) OR Torulopsis glabrata[Title/ Abstract]) OR Torulopsis glabratas[Title/Abstract]) OR ((Torulopsis glabrata[Title/Abstract]) OR Torulopsis glabratas[Title/Abstract])) OR glabrata, Torulopsis[Title/Abstract]) OR Candida tropicalis[MeSH Terms]) OR Candida tropicalis[Title/Abstract]) OR Candida tropicali[Title/Abstract]) OR tropicalis, Candida[Title/Abstract]) OR Candida parapsilosis[MeSH Terms]) OR Candida parapsilosis[Title/ Abstract]) OR C. parapsilosis[Title/Abstract]) OR Candida parapsilosis Complex[Title/Abstract]) OR Candida parapsilosis Group[Title/ Abstract]) OR C. parapsilosis Complex[Title]) OR Candida orthopsilosis[Title/Abstract]) OR C. orthopsilosis[Title/Abstract]) OR Candida metapsilosis[Title/Abstract]) OR C. metapsilosis[Title/Abstract]) OR Candida[MeSH Terms]) OR Candida[Title]) OR Candidas[Title/ Abstract]) OR ((Candida[Title]) OR Candidas[Title/Abstract])) OR Stomatitis, Denture[MeSH Terms]) OR Stomatitis, Denture[Title/ Abstract]) OR Denture Stomatitis[Title/Abstract]) OR ((Stomatitis, Denture[Title/Abstract]) OR Denture Stomatitis[Title/Abstract])) OR Denture Stomatitides[Title/Abstract]) OR Stomatitides, Denture[Title/Abstract]) OR ((Denture Stomatitides[Title/Abstract]) OR Stomatitides, Denture[Title/Abstract])) OR Candida species[Title/Abstract]) OR Candida spp[Title/Abstract])))

\section{\#1 AND \#2}

\#1 TITLE-ABS-KEY (diabetes AND mellitus) OR TITLE-ABS-KEY (diet, AND diabetic) OR TITLE-ABS-KEY (prediabetic AND state) OR TITLE-ABS-KEY (glucose AND intolerance) OR TITLE-ABS-KEY (diabetes AND mellitus, AND type 2 ) OR TITLEABS-KEY (type AND ii AND diabetic) OR TITLE-ABS-KEY (type 2 diabetes AND mellitus) OR TITLE-ABS-KEY (diabetes AND mellitus, AND noninsulin-dependent) OR TITLE-ABS-KEY (diabetes AND mellitus, AND ketosis-resistant) OR TITLE-ABS-KEY (ketosis-resistant AND diabetes AND mellitus) OR TITLE-ABS-KEY (diabetes AND mellitus, AND non AND insulin AND dependent) OR TITLE-ABS-KEY (non-insulin-dependent AND diabetes AND mellitus) OR TITLE-ABS-KEY (diabetes AND mellitus, AND stable) OR TITLE-ABS-KEY ( stable AND diabetes AND mellitus) OR TITLE-ABS-KEY (diabetes AND mellitus, AND type AND ii) OR TITLE-ABS-KEY (niddm) OR TITLE-ABS-KEY (diabetes AND mellitus, AND noninsulin AND dependent ) OR TITLE-ABS-KEY (diabetes AND mellitus, AND maturity-onset) OR TITLE-ABS-KEY (diabetes AND mellitus, AND maturity AND onset) OR TITLE-ABS-KEY (mody) OR TITLE-ABS-KEY (diabetes AND mellitus, AND slow-onset) OR TITLE-ABS-KEY ( slow-onset AND diabetes AND mellitus) OR TITLE-ABS-KEY (noninsulin-dependent AND diabetes AND mellitus) OR TITLEABS-KEY (maturity-onset AND diabetes) OR TITLE-ABS-KEY (type 2 diabetes) OR TITLE-ABS-KEY (adult-onset AND diabetes AND mellitus) OR TITLE-ABS-KEY (diabetes AND mellitus, AND adult AND onset) OR TITLE-ABS-KEY (diabetes AND mellitus, AND type 1 ) OR TITLE-ABS-KEY (diabetes AND mellitus, AND brittle) OR TITLE-ABS-KEY (brittle AND diabetes AND mellitus) OR TITLE-ABS-KEY (diabetes AND mellitus, AND insulin-dependent) OR TITLE-ABS-KEY (insulin-dependent AND diabetes AND mellitus) OR TITLE-ABS-KEY (diabetes AND mellitus, AND juvenile-onset) OR TITLE-ABS-KEY (juvenileonset AND diabetes AND mellitus) OR TITLE-ABS-KEY (diabetes AND mellitus, AND ketosis-prone) OR TITLE-ABS-KEY ( ketosis-prone AND diabetes AND mellitus) OR TITLE-ABS-KEY (juvenile-onset AND diabetes) OR TITLE-ABS-KEY (diabetes AND mellitus, AND type AND i) OR TITLE-ABS-KEY (type 1 diabetes AND mellitus) OR TITLE-ABS-KEY (diabetes AND mellitus, AND sudden-onset) OR TITLE-ABS-KEY (mellitus, AND sudden-onset AND diabetes) OR TITLE-ABS-KEY ( suddenonset AND diabetes AND mellitus) OR TITLE-ABS-KEY (diabetes AND mellitus, AND insulin-dependent, 1 ) OR TITLE-ABSKEY (insulin-dependent AND diabetes AND mellitus 1 ) OR TITLE-ABS-KEY (type 1 diabetes) OR TITLE-ABS-KEY (diabetes, AND type 1 ) OR TITLE-ABS-KEY (iddm) OR TITLE-ABS-KEY (autoimmune AND diabetes)

continue... 
\#2 TITLE-ABS-KEY (candidiasis, AND oral) OR TITLE-ABS-KEY (oral AND candidiases) OR TITLE-ABS-KEY (oral AND candidiasis) OR TITLE-ABS-KEY (thrush) OR TITLE-ABS-KEY (moniliasis, AND oral) OR TITLE-ABS-KEY (moniliases, AND oral) OR TITLE-ABS-KEY (oral AND moniliases) OR TITLE-ABS-KEY (oral AND moniliasis) OR TITLE-ABS-KEY (candida AND albicans) OR TITLE-ABS-KEY (candida AND albican) OR TITLE-ABS-KEY (albicans, AND candida) OR TITLE-ABSKEY (candida AND glabrata) OR TITLE-ABS-KEY (candida AND glabratas) OR TITLE-ABS-KEY (glabratas, AND candida) OR TITLE-ABS-KEY (torulopsis AND glabrata) OR TITLE-ABS-KEY (torulopsis AND glabratas) OR TITLE-ABS-KEY (glabrata, AND torulopsis) OR TITLE-ABS-KEY (candida AND tropicalis) OR TITLE-ABS-KEY (candida AND tropicali) OR TITLE-ABSScopus KEY (tropicalis, AND candida) OR TITLE-ABS-KEY (candida AND parapsilosis) OR TITLE-ABS-KEY (c. AND parapsilosis) OR TITLE-ABS-KEY (candida AND parapsilosis AND complex) OR TITLE-ABS-KEY (candida AND parapsilosis AND group ) OR TITLE-ABS-KEY (c. AND parapsilosis AND complex) OR TITLE-ABS-KEY (candida AND orthopsilosis) OR TITLE-ABSKEY (c. AND orthopsilosis) OR TITLE-ABS-KEY (candida AND metapsilosis) OR TITLE-ABS-KEY (c. AND metapsilosis) OR TITLE-ABS-KEY (candida) OR TITLE-ABS-KEY (candidas) OR TITLE-ABS-KEY (stomatitis, AND denture) OR TITLE-ABS-KEY (denture AND stomatitis) OR TITLE-ABS-KEY (denture AND stomatitides) OR TITLE-ABS-KEY (stomatitides, AND denture) OR TITLE-ABS-KEY (candida AND species) OR TITLE-ABS-KEY (candida AND spp) AND (LIMIT-TO (DOCTYPE, "ar" ))

\section{\#1 AND \#2}

\#1 TS=("Diabetes Mellitus" OR "Diet, Diabetic" OR "Prediabetic State" OR "Glucose Intolerance" OR "Diabetes Mellitus, Type 2" OR "type II diabetic" OR "Diabetes Mellitus, Type 2" OR "Type 2 Diabetes Mellitus" OR "Diabetes Mellitus, Noninsulin-Dependent" OR "Diabetes Mellitus, Ketosis-Resistant" OR "Ketosis-Resistant Diabetes Mellitus" OR "Diabetes Mellitus, Non Insulin Dependent" OR "Non-Insulin-Dependent Diabetes Mellitus" OR "Diabetes Mellitus, Stable" OR "Stable Diabetes Mellitus" OR "Diabetes Mellitus, Type II" OR NIDDM OR "Diabetes Mellitus, Noninsulin Dependent" OR "Diabetes Mellitus, Maturity-Onset" OR "Diabetes Mellitus, Maturity Onset" OR "Maturity-Onset Diabetes Mellitus" OR "MODY" OR "Diabetes Mellitus, Slow-Onset" OR "Slow-Onset Diabetes Mellitus" OR "Noninsulin-Dependent Diabetes Mellitus" OR "Maturity-Onset Diabetes" OR "Type 2 Diabetes" OR "Adult-Onset Diabetes Mellitus" OR "Diabetes Mellitus, Adult Onset" OR "Diabetes Mellitus, Type 1" OR "Diabetes Mellitus, Brittle" OR "Brittle Diabetes Mellitus" OR "Diabetes Mellitus, Insulin-Dependent" OR "Insulin-Dependent Diabetes Mellitus" OR "Diabetes Mellitus, Juvenile-Onset" OR "Juvenile-Onset Diabetes Mellitus" OR "Diabetes Mellitus, Ketosis-Prone" OR "Ketosis-Prone Diabetes Mellitus" OR "Juvenile-

Web of Onset Diabetes" OR "Diabetes Mellitus, Type I" OR "Type 1 Diabetes Mellitus" OR "Diabetes Mellitus, Sudden-Onset" OR "Mellitus,

Science Sudden-Onset Diabetes" OR "Sudden-Onset Diabetes Mellitus" OR "Diabetes Mellitus, Insulin-Dependent, 1" OR "Insulin-Dependent Diabetes Mellitus 1" OR "Type 1 Diabetes" OR "Diabetes, Type 1" OR "IDDM" OR "Autoimmune Diabetes")

\#2 TS= ("Candidiasis, Oral" OR "Oral Candidiasis" OR "Oral Candidiases" OR Thrush OR "Moniliasis, Oral" OR "Moniliases, Oral" OR "Oral Moniliases" OR "Oral Moniliasis" OR "Candida albicans" OR "Candida albican" OR "albicans, Candida" OR "Candida glabrata" OR "Candida glabrata" OR "Candida glabratas" OR "Torulopsis glabrata" OR "Torulopsis glabratas" OR "glabrata, Torulopsis" OR "Candida tropicalis" OR "Candida tropicali" OR "tropicalis, Candida" OR "Candida parapsilosis" OR "C. parapsilosis" OR "Candida parapsilosis Complex" OR "Candida parapsilosis Group" OR "C. parapsilosis Complex" OR

"Candida orthopsilosis" OR "C. orthopsilosis" OR Candida OR Candidas OR "Stomatitis, Denture" OR "Denture Stomatitis" OR "Denture Stomatitides" OR "Stomatitides, Denture" OR "Candida species" OR "Candida spp")

\section{\#1 AND \#2}

\# 1 ("Diabetes Mellitus" OR "Diet, Diabetic" OR "Prediabetic State" OR "Glucose Intolerance" OR "Diabetes Mellitus, Type 2" OR "type II diabetic" OR "Diabetes Mellitus, Type 2" OR "Type 2 Diabetes Mellitus" OR "Diabetes Mellitus, NoninsulinDependent" OR "Diabetes Mellitus, Ketosis-Resistant" OR "Ketosis-Resistant Diabetes Mellitus" OR "Diabetes Mellitus, Non Insulin Dependent" OR "Non-Insulin-Dependent Diabetes Mellitus" OR "Diabetes Mellitus, Stable" OR "Stable Diabetes Mellitus" OR "Diabetes Mellitus, Type II" OR "NIDDM" OR "Diabetes Mellitus, Noninsulin Dependent" OR "Diabetes Mellitus, Maturity-Onset" OR "Diabetes Mellitus, Maturity Onset" OR "Maturity-Onset Diabetes Mellitus" OR "MODY" OR "Diabetes Mellitus, Slow-Onset" OR "Slow-Onset Diabetes Mellitus" OR "Noninsulin-Dependent Diabetes Mellitus" OR "Maturity-Onset Diabetes" OR "Type 2 Diabetes" OR "Adult-Onset Diabetes Mellitus" OR "Diabetes Mellitus, Adult Onset" OR "Diabetes Mellitus, Type 1" OR "Diabetes Mellitus, Brittle" OR "Brittle Diabetes Mellitus" OR "Diabetes Mellitus, Insulin-Dependent" OR "Insulin-Dependent Diabetes Mellitus" OR "Diabetes Mellitus, Juvenile-Onset" OR "Juvenile-Onset Diabetes Mellitus" OR

"Diabetes Mellitus, Ketosis-Prone" OR "Ketosis-Prone Diabetes Mellitus" OR "Juvenile-Onset Diabetes" OR "Diabetes Mellitus, Type I" OR "Type 1 Diabetes Mellitus" OR "Diabetes Mellitus, Sudden-Onset" OR "Mellitus, Sudden-Onset Diabetes" OR

"Sudden-Onset Diabetes Mellitus" OR "Diabetes Mellitus, Insulin-Dependent, 1" OR "Insulin-Dependent Diabetes Mellitus 1" OR "Type 1 Diabetes" OR "Diabetes, Type 1" OR "IDDM" OR "Autoimmune Diabetes")

\#2 ("Candidiasis, Oral" OR "Oral Candidiasis" OR "Oral Candidiases" OR Thrush OR "Moniliasis, Oral" OR "Moniliases, Oral" OR "Oral Moniliases" OR "Oral Moniliasis" OR "Candida albicans" OR "Candida albican" OR "albicans, Candida" OR "Candida glabrata" OR "Candida glabrata" OR "Candida glabratas" OR "Torulopsis glabrata" OR "Torulopsis glabratas" OR "glabrata, Torulopsis" OR "Candida tropicalis" OR "Candida tropicali" OR "tropicalis, Candida" OR "Candida parapsilosis" OR "C. parapsilosis" OR "Candida parapsilosis Complex" OR "Candida parapsilosis Group" OR "C. parapsilosis Complex" OR "Candida orthopsilosis" OR "C. orthopsilosis" OR Candida OR Candidas OR "Stomatitis, Denture" OR "Denture Stomatitis" OR "Denture Stomatitides" OR "Stomatitides, Denture" OR “Candida species" OR "Candida spp") 
\# $1 \mathrm{MeSH}$ descriptor: [Diabetes Mellitus] explode all trees

\#2 Diabetes Mellitus OR Diet, Diabetic OR Prediabetic State OR Glucose Intolerance

\#3 MeSH descriptor: [Diabetes Mellitus, Type 2] explode all trees

\#4 Diabetes Mellitus, Type 2 OR type II diabetic OR Diabetes Mellitus, Type 2 OR Type 2 Diabetes Mellitus OR Diabetes Mellitus, Noninsulin-Dependent OR Diabetes Mellitus, Ketosis-Resistant OR Ketosis-Resistant Diabetes Mellitus OR Diabetes Mellitus, Non Insulin Dependent OR Non-Insulin-Dependent Diabetes Mellitus OR Diabetes Mellitus, Stable OR Stable Diabetes Mellitus OR Diabetes Mellitus, Type II OR NIDDM OR Diabetes Mellitus, Noninsulin Dependent OR Diabetes Mellitus, MaturityOnset OR Diabetes Mellitus, Maturity Onset OR Maturity-Onset Diabetes Mellitus OR MODY OR Diabetes Mellitus, Slow-Onset OR Slow-Onset Diabetes Mellitus OR Noninsulin-Dependent Diabetes Mellitus OR Maturity-Onset Diabetes OR Type 2 Diabetes

OR Adult-Onset Diabetes Mellitus OR Diabetes Mellitus, Adult Onset

\#5 MeSH descriptor: [Diabetes Mellitus, Type 1] explode all trees

\#6 Diabetes Mellitus, Type 1 OR Diabetes Mellitus, Brittle OR Brittle Diabetes Mellitus OR Diabetes Mellitus, Insulin-Dependent OR Insulin-Dependent Diabetes Mellitus OR Diabetes Mellitus, Juvenile-Onset OR Juvenile-Onset Diabetes Mellitus OR

Diabetes Mellitus, Ketosis-Prone OR Ketosis-Prone Diabetes Mellitus OR Juvenile-Onset Diabetes OR Diabetes Mellitus, Type I OR Type 1 Diabetes Mellitus OR Diabetes Mellitus, Sudden-Onset OR Mellitus, Sudden-Onset Diabetes OR Sudden-Onset Diabetes Mellitus OR Diabetes Mellitus, Insulin-Dependent, 1 OR Insulin-Dependent Diabetes Mellitus 1 OR Type 1 Diabetes OR Diabetes, Type 1 OR IDDM OR Autoimmune Diabetes

\#7 \#1 or \#2 or \#3 or \#4 or \#5 or \#6

\#8 MeSH descriptor: [Candidiasis, oral] explode all trees

Cochrane Library

\#9 Candidiasis, Oral OR Oral Candidiases OR Oral Candidiasis OR Thrush OR Moniliasis, Oral OR Moniliases, Oral OR Oral Moniliases OR Oral Moniliasis

\# $10 \mathrm{MeSH}$ descriptor: [Candida albicans] explode all trees

\# 11 Candida albicans OR Candida albican OR albicans, Candida

\# $12 \mathrm{MeSH}$ descriptor: [Candida glabrata] explode all trees

\# 13 Candida glabrata OR Candida glabratas OR Torulopsis glabrata OR Torulopsis glabratas OR glabrata, Torulopsis

\# $14 \mathrm{MeSH}$ descriptor: [Candida tropicalis] explode all trees

\# 15 Candida tropicalis OR Candida tropicali OR tropicalis, Candida

\# $16 \mathrm{MeSH}$ descriptor: [Candida parapsilosis] explode all trees

\# 17 Candida parapsilosis OR C. parapsilosis OR Candida parapsilosis Complex OR Candida parapsilosis Group OR C. parapsilosis Complex OR Candida orthopsilosis OR C. orthopsilosis OR Candida metapsilosis OR C. metapsilosis

\# $18 \mathrm{MeSH}$ descriptor: [Candida] explode all trees

\# 19 Candida OR Candidas

\#20 MeSH descriptor: [Stomatitis, denture] explode all trees

\#21 Stomatitis, Denture OR Denture Stomatitis OR Denture Stomatitides OR Stomatitides, Denture OR Candida species OR Candida spp \#22 \#8 or \#9 or \#10 or \#11 or \#12 or \#13 or \#14 or \#15 or \#16 or \#17 or \#18 or \#19 or \#20 or \#21

\#23 \#7 and \#22

\# 1 (tw:((tw:(Diabetes Mellitus)) OR (tw:(Diet, Diabetic)) OR (tw:(Prediabetic State)) OR (tw:(Glucose Intolerance)) OR (tw: (Diabetes Mellitus, Type 2)) OR (tw:(type II diabetic)) OR (tw:(Diabetes Mellitus, Type 2)) OR (tw:(Type 2 Diabetes Mellitus)) OR (tw:(Diabetes Mellitus, Noninsulin-Dependent)) OR (tw:(Diabetes Mellitus, Ketosis-Resistant)) OR (tw:(Ketosis-Resistant Diabetes Mellitus)) OR (tw:(Diabetes Mellitus, Non Insulin Dependent)) OR (tw:(Non-Insulin-Dependent Diabetes Mellitus)) OR (tw:(Diabetes Mellitus,

Stable)) OR (tw:(Stable Diabetes Mellitus)) OR (tw:(Diabetes Mellitus, Type II)) OR (tw:(NIDDM)) OR (tw:(Diabetes Mellitus, Noninsulin Dependent)) OR (tw:(Diabetes Mellitus, Maturity-Onset)) OR (tw:(Diabetes Mellitus, Maturity Onset)) OR (tw:(MaturityOnset Diabetes Mellitus)) OR (tw:(MODY)) OR (tw:(Diabetes Mellitus, Slow-Onset)) OR (tw:(Slow-Onset Diabetes Mellitus)) OR (tw:(Noninsulin-Dependent Diabetes Mellitus)) OR (tw:(Maturity-Onset Diabetes)) OR (tw:(Type 2 Diabetes)) OR (tw:(Adult-Onset Diabetes Mellitus)) OR (tw:(Diabetes Mellitus, Adult Onset)) OR (tw:(Diabetes Mellitus, Type 1)) OR (tw:(Diabetes Mellitus, Brittle)) OR (tw: (Brittle Diabetes Mellitus)) OR (tw:(Diabetes Mellitus, Insulin-Dependent)) OR (tw:(Insulin-Dependent Diabetes Mellitus)) OR (tw:(Diabetes Mellitus, Juvenile-Onset)) OR (tw:(Juvenile-Onset Diabetes Mellitus)) OR (tw:(Diabetes Mellitus, Ketosis-Prone)) OR (tw:(Ketosis-Prone Diabetes Mellitus)) OR (tw:(Juvenile-Onset Diabetes)) OR (tw:(Diabetes Mellitus, Type I)) OR (tw:(Type 1

Diabetes Mellitus)) OR (tw:(Diabetes Mellitus, Sudden-Onset)) OR (tw:(Mellitus, Sudden-Onset Diabetes)) OR (tw:(Sudden-Onset Diabetes Mellitus)) OR (tw:(Diabetes Mellitus, Insulin-Dependent, 1)) OR (tw:(Insulin-Dependent Diabetes Mellitus 1)) OR (tw:(Type 1 Diabetes)) OR (tw:(Diabetes, Type 1)) OR (tw:(IDDM)) OR (tw:(Autoimmune Diabetes))))

continue... 
\#2 (tw:((tw:(Candidiasis, Oral)) OR (tw:(Oral Candidiases)) OR (tw:(Oral Candidiasis)) OR (tw:(Thrush)) OR (tw:(Moniliasis, Oral)) OR (tw:(Moniliases, Oral)) OR (tw:(Oral Moniliases)) OR (tw:(Oral Moniliasis)) OR (tw:(Candida albicans)) OR

(tw:(Candida albican)) OR (tw:(albicans, Candida)) OR (tw:(Candida glabrata)) OR (tw:(Candida glabrata)) OR (tw: (Candida glabratas)) OR (tw:(Torulopsis glabrata)) OR (tw:(Torulopsis glabratas)) OR (tw: (glabrata, Torulopsis)) OR (tw: (Candida $\begin{array}{ll}\text { tropicalis)) OR (tw: (Candida tropicali)) OR (tw:(tropicalis, Candida)) OR (tw:(Candida parapsilosis)) OR (tw: (C. parapsilosis)) OR } \\ \text { Lilacs } & \text { (tw:(Candida parapsilosis Complex)) OR (tw:(Candida parapsilosis Group)) OR (tw:(C. parapsilosis Complex)) OR (tw:(Candida }\end{array}$ orthopsilosis)) OR (tw:(C. orthopsilosis)) OR (tw:(Candida metapsilosis)) OR (tw:(C. metapsilosis)) OR (tw:(Candida)) OR (tw: (Candidas)) OR (tw:(Stomatitis, Denture)) OR (tw:(Denture Stomatitis)) OR (tw:(Denture Stomatitides)) OR (tw:(Stomatitides, Denture)) OR (tw:(Candida species)) OR (tw:(Candida spp))))

\section{\#1 AND \#2}

\#1 diabetes:ti,ab,kw AND mellitus:ti,ab,kw OR (diet,:ti,ab,kw AND diabetic:ti,ab,kw) OR (prediabetic:ti,ab, kw AND state:ti, ab, kw) OR (glucose:ti,ab,kw AND intolerance:ti,ab,kw) OR (diabetes:ti,ab, kw AND mellitus, :ti,ab, kw AND 'type 2':ti,ab, kw) OR (type:ti,ab, kw AND ii:ti,ab,kw AND diabetic:ti,ab,kw) OR ('type 2 diabetes':ti,ab,kw AND mellitus:ti,ab,kw) OR (diabetes:ti, ab, kw AND mellitus, :ti, ab, kw AND 'noninsulin dependent':ti, ab,kw) OR (diabetes:ti, ab, kw AND mellitus, :ti, ab, kw AND 'ketosis resistant':ti, ab,kw) OR ('ketosis resistant':ti,ab,kw AND diabetes:ti, ab, kw AND mellitus:ti,ab,kw) OR (diabetes:ti,ab,kw AND mellitus,:ti,ab,kw AND non:ti,ab,kw AND insulin:ti,ab,kw AND dependent:ti,ab,kw) OR ('non insulin dependent':ti,ab,kw AND diabetes:ti,ab, kw AND mellitus:ti,ab,kw) OR (diabetes:ti, ab,kw AND mellitus, :ti,ab,kw AND stable:ti,ab,kw) OR (stable:ti,ab,kw AND diabetes:ti,ab,kw AND mellitus:ti,ab,kw) OR

(diabetes:ti,ab,kw AND mellitus,:ti,ab,kw AND type:ti,ab,kw AND ii:ti,ab,kw) OR niddm:ti,ab,kw OR (diabetes:ti,ab,kw AND mellitus, :ti,ab, kw AND noninsulin:ti,ab,kw AND dependent:ti,ab,kw) OR (diabetes:ti,ab,kw AND mellitus,:ti,ab,kw AND 'maturity onset':ti,ab,kw) OR (diabetes:ti,ab,kw AND mellitus, :ti, ab, kw AND maturity:ti, ab, kw AND onset:ti, ab, kw) OR mody:ti,ab, kw OR (diabetes:ti,ab,kw AND mellitus,:ti, ab,kw AND 'slow onset':ti,ab,kw) OR ('slow onset':ti, ab, kw AND diabetes:ti,ab,kw AND mellitus:ti,ab,kw) OR ('noninsulin dependent':ti,ab,kw AND diabetes:ti,ab,kw AND mellitus:ti,ab,kw)

OR ('maturity onset':ti,ab,kw AND diabetes:ti,ab,kw) OR 'type 2 diabetes':ti,ab,kw OR ('adult onset':ti,ab, kw AND diabetes:ti, ab, kw AND mellitus:ti, ab,kw) OR (diabetes:ti, ab, kw AND mellitus,:ti,ab, kw AND adult:ti, ab, kw AND onset:ti, ab, kw) OR (diabetes:ti,ab,kw AND mellitus, :ti, ab,kw AND 'type 1':ti,ab, kw) OR (diabetes:ti,ab, kw AND mellitus, :ti, ab, kw AND brittle:ti,ab,kw) OR (brittle:ti,ab,kw AND diabetes:ti,ab,kw AND mellitus:ti,ab,kw) OR (diabetes:ti,ab,kw AND mellitus,:ti,ab, kw AND 'insulin dependent':ti,ab,kw) OR ('insulin dependent':ti,ab,kw AND diabetes:ti, ab,kw AND mellitus:ti,ab,kw) OR (diabetes:ti,ab,kw AND mellitus,:ti, ab, kw AND 'juvenile onset' :ti,ab, kw) OR ('juvenile onset':ti, ab, kw AND diabetes:ti, ab, kw AND mellitus:ti,ab, kw) OR (diabetes:ti,ab,kw AND mellitus, :ti, ab, kw AND 'ketosis prone':ti,ab, kw) OR ('ketosis prone':ti,ab,kw AND diabetes:ti,ab,kw AND mellitus:ti,ab,kw) OR ('juvenile onset':ti,ab, kw AND diabetes:ti,ab, kw) OR (diabetes:ti,ab, kw AND mellitus, :ti,ab,kw AND type:ti,ab,kw AND i:ti,ab,kw) OR ('type 1 diabetes':ti,ab,kw AND mellitus:ti,ab, kw) OR (diabetes:ti, ab, kw AND mellitus, :ti,ab,kw AND 'sudden onset':ti,ab,kw) OR (mellitus, :ti, ab, kw AND 'sudden onset':ti,ab, kw AND diabetes:ti,ab,kw) OR ('sudden onset':ti,ab, kw AND diabetes:ti,ab, kw AND mellitus:ti,ab,kw) OR (diabetes:ti, ab, kw AND mellitus,:ti,ab, kw AND 'insulin-dependent, 1':ti,ab,kw) OR ('insulin dependent':ti,ab, kw AND diabetes:ti,ab,kw AND 'mellitus $\left.l^{\prime}: t i, a b, k w\right)$ OR 'type 1 diabetes':ti,ab,kw OR (diabetes,:ti,ab, kw AND 'type $l^{\prime}: t i, a b, k w$ ) OR iddm:ti,ab,kw OR (autoimmune:ti,ab,kw AND diabetes:ti,ab,kw)

\#2 candidiasis,:ti,ab,kw AND oral:ti,ab, kw OR (oral:ti,ab, kw AND candidiases:ti,ab,kw) OR (oral:ti,ab,kw AND candidiasis:ti,ab,kw) OR thrush:ti,ab,kw OR (moniliasis,:ti,ab,kw AND oral:ti,ab,kw) OR (moniliases, :ti,ab,kw AND oral:ti,ab, kw) OR (oral:ti,ab,kw AND moniliases:ti,ab,kw) OR (oral:ti,ab,kw AND moniliasis:ti,ab,kw) OR (candida:ti,ab, kw AND albicans:ti,ab,kw) OR (candida:ti,ab,kw AND albican:ti,ab,kw) OR (albicans, :ti,ab,kw AND candida:ti,ab,kw) OR (candida:ti,ab, kw AND glabrata:ti,ab,kw) OR (candida:ti,ab, kw AND glabratas:ti,ab,kw) OR (glabratas, :ti,ab, kw AND candida:ti,ab,kw) OR (torulopsis:ti,ab,kw AND glabrata:ti,ab,kw) OR (†orulopsis:ti,ab,kw AND glabratas:ti,ab,kw) OR (glabrata, :ti,ab,kw AND torulopsis:ti,ab,kw) OR (candida:ti,ab,kw AND tropicalis:ti,ab,kw) OR (candida:ti,ab, kw AND tropicali:ti,ab,kw) OR (tropicalis,:ti,ab, kw AND candida:ti,ab,kw) OR (candida:ti,ab,kw AND parapsilosis:ti,ab,kw) OR (c.:ti,ab,kw AND parapsilosis:ti,ab,kw) OR (candida:ti,ab,kw AND parapsilosis:ti,ab,kw AND complex:ti,ab,kw) OR (candida:ti,ab,kw AND parapsilosis:ti,ab,kw AND group:ti,ab,kw) OR (c.:ti,ab, kw AND parapsilosis:ti,ab,kw AND complex:ti,ab,kw) OR (candida:ti,ab,kw AND orthopsilosis:ti,ab,kw) OR (c.:ti,ab,kw AND orthopsilosis:ti,ab,kw) OR (candida:ti,ab, kw AND metapsilosis:ti,ab,kw) OR (c.:ti,ab,kw AND metapsilosis:ti,ab,kw) OR candida:ti,ab,kw OR candidas:ti,ab,kw OR (stomatitis,:ti,ab,kw AND denture:ti,ab,kw) OR (denture:ti,ab,kw AND stomatitis:ti,ab,kw) OR (denture:ti,ab, kw AND stomatitides:ti,ab,kw) OR (stomatitides, :ti, ab,kw AND denture:ti,ab,kw) OR (candida:ti,ab,kw AND species:ti,ab,kw) OR (candida:ti,ab,kw AND spp:ti,ab,kw)

abstract did not allow for proper exclusion, the full-text publication was also read to mitigate any doubts. A third examiner solved any disagreement between the two reviewers.

\section{Data extraction and quality assessment}

Before the analysis, the two examiners were trained on data extraction and quality assessment. Data were extracted to a single spreadsheet, 
which included information on the study design, population, gender, sample size, method used to verify the absence or presence of diabetes mellitus, and the number of cases and rate of oral candidiasis or denture stomatitis in diabetic and non-diabetic patients.

After data extraction, a qualitative synthesis was performed following the recommendations of Fowkes and Fulton. ${ }^{23}$ This tool is a guideline used in the field of dentistry ${ }^{24,25}$ for the critical analysis of articles, in which certain items are investigated, such as study design, sample representativity, validity, reproducibility, losses, and bias. Two examiners proceeded with classifying articles and making a checklist (Table 2). For each item, two examiners attributed scores that represented a major problem $(++)$, minor problem $(+)$, no problem $(0)$, or not applicable (NA). Sample size, sample selection, blindness, and research instruments were considered essential criteria for the quality assessment of included studies. Summary question classification on the risk of bias, confounding factors, and the chance of results occurring by chance was implemented to generate categories of no, low, and high risk of bias in studies.

When examiners disagreed, a third researcher conducted the assessment and proposed a consensus. In cases where articles did not contain the required information or were incomplete, at least three emails were sent to the authors within a 5-week period in an attempt to obtain the required information.

\section{Quantitative synthesis (Meta-analysis)}

The extracted data were analyzed using RevMan software (Review Manager, version 5.3, The Cochrane Collaboration; Copenhagen, Denmark) to assess the relationship between the disease (oral candidiasis or denture stomatitis) and diabetes mellitus. The prevalence of candida infection (events) and the total number of individuals in the case (with diabetes mellitus) and control (without diabetes mellitus) groups were included to calculate the Odds Ratio (OR), with a $95 \%$ confidence interval (CI). Two meta-analyses were performed on studies evaluating patients with: a) oral candidiasis and b) denture stomatitis. A fixed-effect model was used. ${ }^{26}$

\section{Assessment of certainty of the evidence}

The quality of the evidence (certainty in the estimates of effect) was determined for the outcome using the Grading of Recommendations Assessment, Development and Evaluation (GRADE) ${ }^{27}$ approach, in which observational studies start as low evidence. The quality of, or certainty in, evidence decreases to very low if serious or very serious issues are described related to the risk of bias. Such issues include inconsistency, indirectness, imprecision, and publication bias. In addition, the quality of the evidence could be upgraded if the magnitude of the effect is large or very large, or if the effect of all plausible confounding factors reduces the effect, or suggests a spurious effect. Thus, the quality of evidence could vary from very low to high.

\section{Results}

A total of 6034 articles were identified. After removing duplicates, 2794 papers remained in the analysis. Subsequently, the titles and abstracts were read, and 49 articles were selected for fulltext reading. After full-text reading, 14 studies were excluded because the selected patients had confounding factors (e.g., smoking, alcohol consumption, immunosuppression, and other comorbidities). A further 28 studies were also excluded, because they had different outcomes. Finally, seven articles were selected for qualitative and quantitative synthesis (meta-analysis). Although hand-searching was performed, no articles were retrieved through this process. Figure 1 presents the flowchart of the study selection process.

\section{Data extraction and quality assessment}

The methodological analysis of the included articles is presented in Table 3. Six articles were classified as cross-sectional studies ${ }^{12,28,29,30,31,32}$ and one was classified as a cohort study. ${ }^{11}$ This study ${ }^{11}$ was included because the results on oral candidiasis were shown as cross-sectional data. The articles were published between 1996 and 2017, with the population being composed of adults of both genders. The group of exposed patients was diagnosed with diabetes, while the control group was composed of healthy patients. 
Oral candidiasis and denture stomatitis in diabetic patients: Systematic review and meta-analysis

Table 2. Classification of the articles using a checklist based on Fowkes and Fulton (1991).

\begin{tabular}{|c|c|c|}
\hline Guideline & Checklist & Established criteria \\
\hline \multirow{11}{*}{$\begin{array}{l}\text { Study sample } \\
\text { representative? }\end{array}$} & & 0: Participants of the same center and homogeneity of the sample \\
\hline & Source of sample & + Participants from different centers \\
\hline & & ++ Participants from different centers and imbalance in the sample \\
\hline & & 0: Samples paired by gender, age and sample size \\
\hline & Sampling method & $+:$ It stopped the sample only in some aspects \\
\hline & & ++ : Did not match or did not make it clear the pairing of samples \\
\hline & & 0: The study performed a sample calculation \\
\hline & Sample size & + : No sample calculation was performed, but the sample is representative \\
\hline & & ++ : Did not do sample calculation and sample is not representative \\
\hline & Entry criteria/ & $\begin{array}{l}\text { 0: Clearly defined inclusion and exclusion criteria, excluding smokers, alcoholics, } \\
\text { immunocompromised and other comorbidities }\end{array}$ \\
\hline & exclusions & $\begin{array}{c}++: \text { The authors stated that the patients were exclusively diabetic, but it was not } \\
\text { mentioned if there were excluded smokers, alcoholics, immunocompromised, and } \\
\text { other comorbidities }\end{array}$ \\
\hline \multirow{9}{*}{ Control group accetable? } & \multirow{3}{*}{ Definition of controls } & 0: Adequate criteria for inclusion of controls (pairing with the exposed group) \\
\hline & & + : Partial matching of criteria for controls \\
\hline & & $++:$ Not matching or not defined \\
\hline & \multirow{3}{*}{ Source of controls } & 0: Participants of the same center and homogeneity of the sample \\
\hline & & + Participants from different centers \\
\hline & & ++ Participants from different centers and imbalance in the sample \\
\hline & \multirow{3}{*}{$\begin{array}{l}\text { Matching / } \\
\text { randomisation }\end{array}$} & $\begin{array}{l}\text { 0: If the control group is paired with experimental (exposed) in gender, age, sample } \\
\text { size and if there was randomization }\end{array}$ \\
\hline & & $+:$ There was pairing in some aspects; There was no matching by sample size \\
\hline & & $++:$ Not matched or not clear. \\
\hline \multirow{20}{*}{$\begin{array}{l}\text { Quality of measurements } \\
\text { and outcomes? }\end{array}$} & \multirow{2}{*}{ Validity } & 0: The study uses validated diagnostic method \\
\hline & & ++ : Does not use previously validated method \\
\hline & \multirow{3}{*}{ Reproducibility } & 0: Calibrated calibrator (with kappa value) \\
\hline & & +: Expert opinion or evaluator calibrated without kappa \\
\hline & & ++ : Did not use expert opinion or calibrated evaluator \\
\hline & \multirow{3}{*}{ Blindness } & 0 : Examiner is blind \\
\hline & & $+:$ There is only blinding of the statistician \\
\hline & & $++:$ There is no type of blinding \\
\hline & \multirow{3}{*}{ Quality control } & 0: More than one valued calibrator with Kappa value \\
\hline & & +: Two calibrated raters (no Kappa value) or one calibrated rater (Kappa value) \\
\hline & & ++ : An uncalibrated evaluator \\
\hline & \multirow{3}{*}{ Missing data } & 0: no data loss \\
\hline & & + : data loss less than $20 \%$ \\
\hline & & $++:$ data loss greater than $20 \%$ \\
\hline & \multirow{3}{*}{ Confounding factors } & 0 : there is no confounding factor \\
\hline & & $+:$ is there any confounding factor \\
\hline & & $++:$ there are serious confounding factors \\
\hline & \multirow{3}{*}{$\begin{array}{l}\text { Distortion reduced by } \\
\text { analysis }\end{array}$} & 0: no distortion or analysis was adjusted for distortions \\
\hline & & $+:$ partially adjusted analysis to reduce confounding \\
\hline & & $++:$ unadjusted analysis \\
\hline
\end{tabular}




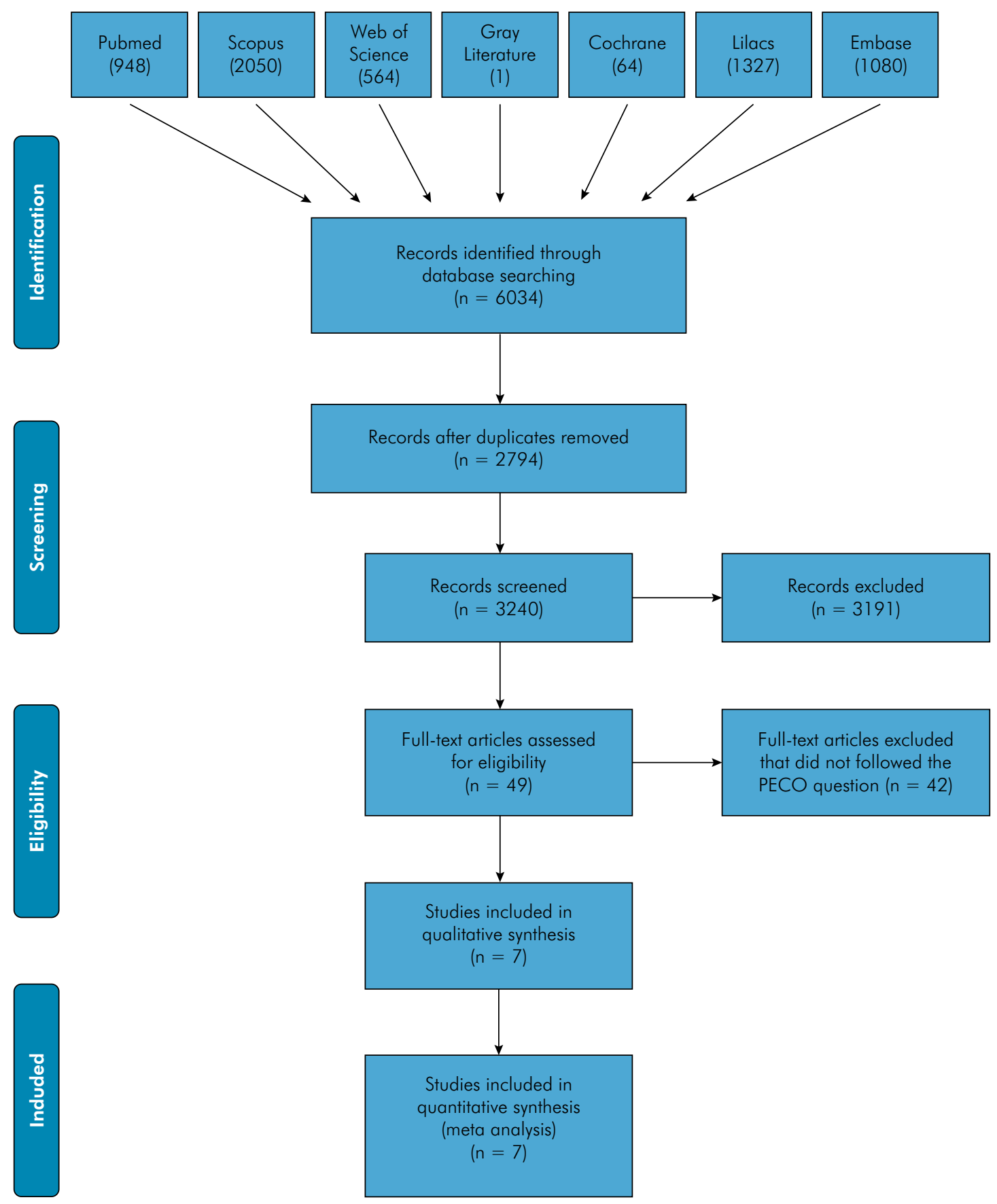

Figure 1. Flow diagram of the literature search based on the PRISMA statement.

Regarding sampling, four studies ${ }^{12,28,30,31}$ recruited participants from different centers (sample sources), which was considered a minor problem $(+)$. Concerning the sample matching, three studies ${ }^{11,12,30}$ did not match the samples with sample size, which we considered as a minor problem $(+)$. Moreover, two studies ${ }^{28,32} \mathrm{did}$ not match the samples with respect to the gender and age of the participants, which was considered a major problem $(++)$.

In most studies, ${ }^{11,12,28,30,32}$ sample calculations were not performed. Thus, to verify whether the sample used was sufficient, we performed 
Oral candidiasis and denture stomatitis in diabetic patients: Systematic review and meta-analysis

Table 3. Quality assessment based on Fowkes and Fulton (1991).

\begin{tabular}{|c|c|c|c|c|c|c|c|c|}
\hline Guideline & Checklist & $\begin{array}{c}\text { Al-Maweri et al., } \\
2013^{29}\end{array}$ & $\begin{array}{c}\text { DorockaBobkowska } \\
\text { et al., 1996.12 }\end{array}$ & $\begin{array}{c}\text { Radović et al., } \\
2014^{30}\end{array}$ & $\begin{array}{l}\text { Saini et al., } \\
2010^{31}\end{array}$ & $\begin{array}{l}\text { Bissong et al., } \\
2015^{28}\end{array}$ & $\begin{array}{l}\text { Trentin et al., } \\
2017^{32}\end{array}$ & $\begin{array}{l}\text { Obradović et al., } \\
\qquad 2011^{11}\end{array}$ \\
\hline \multirow{4}{*}{$\begin{array}{l}\text { Study design } \\
\text { appropriate to } \\
\text { objectives? }\end{array}$} & Cross-sectional & $x$ & $x$ & $x$ & $x$ & $x$ & $\mathrm{x}$ & \multirow{4}{*}{$\mathrm{x}$} \\
\hline & Cohort & & & & & & & \\
\hline & Controlled trial & & & & & & & \\
\hline & Cause control & & & & & & & \\
\hline \multirow{5}{*}{$\begin{array}{l}\text { Study sample } \\
\text { representative? }\end{array}$} & Source of sample & 0 & + & + & + & + & 0 & 0 \\
\hline & Sampling method & 0 & + & ++ & 0 & ++ & + & + \\
\hline & Sample size & 0 & + & + & 0 & + & + & + \\
\hline & $\begin{array}{l}\text { Entry criteria/ } \\
\text { exclusions }\end{array}$ & 0 & 0 & ++ & 0 & ++ & ++ & ++ \\
\hline & Non-respondents & NA & NA & NA & NA & NA & NA & NA \\
\hline \multirow{4}{*}{$\begin{array}{l}\text { Control group } \\
\text { accetable? }\end{array}$} & $\begin{array}{l}\text { Definition of } \\
\text { controls }\end{array}$ & 0 & + & 0 & 0 & + & + & ++ \\
\hline & $\begin{array}{l}\text { Source of } \\
\text { controls }\end{array}$ & 0 & + & 0 & 0 & 0 & 0 & 0 \\
\hline & $\begin{array}{l}\text { Matching / } \\
\text { randomisation }\end{array}$ & + & ++ & ++ & 0 & ++ & ++ & + \\
\hline & $\begin{array}{c}\text { Comparable } \\
\text { characteristics }\end{array}$ & 0 & + & + & 0 & ++ & ++ & + \\
\hline \multirow{4}{*}{$\begin{array}{l}\text { Quality of } \\
\text { measurements } \\
\text { and outcomes? }\end{array}$} & Validity & 0 & 0 & 0 & 0 & ++ & ++ & ++ \\
\hline & Reproducibility & + & ++ & + & + & ++ & 0 & ++ \\
\hline & Blindness & ++ & ++ & ++ & ++ & ++ & ++ & ++ \\
\hline & Quality control & ++ & ++ & + & ++ & ++ & 0 & ++ \\
\hline \multirow{4}{*}{ Completeness? } & Compliance & NA & NA & NA & NA & NA & NA & NA \\
\hline & Drop outs & NA & NA & NA & NA & NA & NA & NA \\
\hline & Deaths & NA & NA & NA & NA & NA & NA & NA \\
\hline & Missing data & 0 & 0 & + & 0 & 0 & 0 & 0 \\
\hline \multirow{5}{*}{$\begin{array}{l}\text { Distorting } \\
\text { influences? }\end{array}$} & $\begin{array}{l}\text { Extraneous } \\
\text { treatments }\end{array}$ & NA & NA & NA & NA & NA & NA & NA \\
\hline & Contamination & NA & NA & NA & NA & NA & NA & NA \\
\hline & $\begin{array}{l}\text { Changes over } \\
\text { time }\end{array}$ & NA & NA & NA & NA & NA & NA & NA \\
\hline & $\begin{array}{l}\text { Confounding } \\
\text { factors }\end{array}$ & 0 & 0 & ++ & 0 & ++ & ++ & ++ \\
\hline & $\begin{array}{l}\text { Distortion reduced } \\
\text { by analysis }\end{array}$ & 0 & ++ & ++ & 0 & ++ & ++ & ++ \\
\hline \multirow{3}{*}{$\begin{array}{l}\text { Summary } \\
\text { questions }\end{array}$} & $\begin{array}{l}\text { Bias - Are the } \\
\text { results } \\
\text { erroneously } \\
\text { biased in a } \\
\text { certain direction? }\end{array}$ & No & Yes & No & No & Yes & Yes & Yes \\
\hline & $\begin{array}{l}\text { Confounding } \\
\text { - Are there } \\
\text { any serious } \\
\text { confounding or } \\
\text { other distortin } \\
\text { influences? }\end{array}$ & No & No & Yes & No & Yes & Yes & Yes \\
\hline & $\begin{array}{l}\text { Chance - Is it } \\
\text { likely that the } \\
\text { results occurred } \\
\text { by chance? }\end{array}$ & No & No & No & No & Yes & Yes & Yes \\
\hline
\end{tabular}

0: no problem; + minor problem; ++major problem; NA: not applicable. 
sample calculations based on the results of a previous studies. ${ }^{33,34}$ A calculated sample size of 39 participants was sufficient to detect a $20 \%$ difference in prevalence between groups, with a power of $80 \%$ ( $\alpha=0.05 \%$ ). Thus, although sample calculations were not performed in these studies, sample size was considered sufficient.

Inclusion and exclusion criteria were not followed

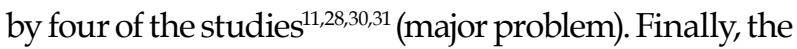
articles that could include confounding factors and the analysis of the results were not stratified or adjusted, which was classified as a major problem (++). This risk of bias was found in six studies, ${ }^{11,12,28,30,31,32}$ showing that the biggest problem is a lack of comparable characteristics between groups.

Other criteria included the method used to evaluate the outcomes. All studies used a clinical examination of the oral cavity, and verified the presence or absence of oral candidiasis or denture stomatitis. Two studies ${ }^{29,31}$ used the parameters established by the World Health Organization, while two others ${ }^{12,30}$ used the Newton classification. The other studies did not report any parameter for clinical evaluation, which was classified as a major problem because the study is not reproducible. Six studies ${ }^{11,12,28,30,31,32}$ did not use calibrated examiners or specialists. Another key point was that the evaluators were not blinded in all studies, representing a major problem $(++)$.

Table 4 presents the characteristics of the studies data collected from them. Three articles showed as oral candidiasis as the outcome ${ }^{11,28,32}$, while four ${ }^{12,29,30,31}$ showed denture stomatitis as the outcome. The prevalence of oral candidiasis ranged from $6.8 \%{ }^{32}$ to $31 \%{ }^{11}$ in patients with diabetes mellitus, and ranged from $2 \%{ }^{11}$ to $14.1 \%^{32}$ for controls. The prevalence of denture stomatitis among diabetic individuals ranged from $10.7 \%^{31}$ to $61.1 \%^{30}$, and ranged from $6.2 \%^{31}$ to $38.1 \%^{30}$ for controls.

\section{Meta-analysis and certainty of the evidence}

All seven studies were included in the quantitative

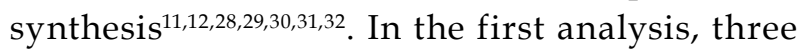
studies ${ }^{11,28,32}$ were included evaluating oral candidiasis as the outcome. This analysis showed that diabetics patients $(n=365)$ had a similar chance of developing oral candidiasis compared to non-diabetic patients ( $\mathrm{n}=286)\left(\mathrm{OR} 1.40\right.$ [0.96; 2.04], $\left.\mathrm{p}=0.08, \mathrm{I}^{2}=94 \%\right)$ (Figure 2). However, these results had very low certainty of evidence, due to serious problems in the risk of bias, and very serious problems with respect to inconsistency and imprecision (Table 5).

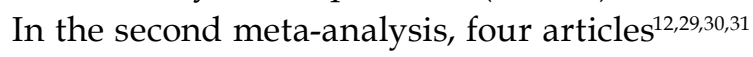
showed denture stomatitis outcome. This analysis showed that diabetic patients $(n=923)$ had a higher chance of denture stomatitis compared to healthy patients $(\mathrm{n}=911)(\mathrm{OR} 1.92[1.42,2.59] \mathrm{p}<0.0001$, $\mathrm{I}^{2}=0 \%$ ) (Figure 3 ). However, these results had very low certainty of evidence, due to serious and very serious problems in indirectness and imprecision, respectively (Table 5).

\section{Discussion}

Previous studies demonstrated a higher prevalence of oral candidiasis and denture stomatitis in diabetic patients. ${ }^{17,18,19,20}$ However, these studies had confounding factors, including tobacco smoking, daily alcohol consumption, and the intake of medication, which are also risk factors of oral candidiasis and denture stomatitis. Thus, to generate a summary of reliable evidence, this systematic review and metaanalysis included studies with no confounding factors. Thus, when compared to non-diabetic individuals, diabetic patients had similar chances of developing oral candidiasis, and a greater chance of developing denture stomatitis.

In our systematic review, the prevalence of oral candidiasis ranged from $6.8 \%{ }^{32}$ to $31 \%{ }^{11}$ in patients with diabetes mellitus, whereas the prevalence of denture stomatitis ranged from $10.7 \%{ }^{31}$ to $61.1 \%^{30}$ in these same patients. Previous studies ${ }^{11,12,30}$ that obtained a higher prevalence of both diseases (i.e., > 30\% in diabetic individuals) might have been subject to bias in participant selection, whereby participants were not paired and had heterogeneous characteristics. Thus, the data might have been overestimated, resulting in a higher prevalence of recorded oral lesions. Hence, future studies should include individuals paired by gender, age, similar systemic and oral conditions, and sample size. The paired samples process provides reliable and reproducible results. 


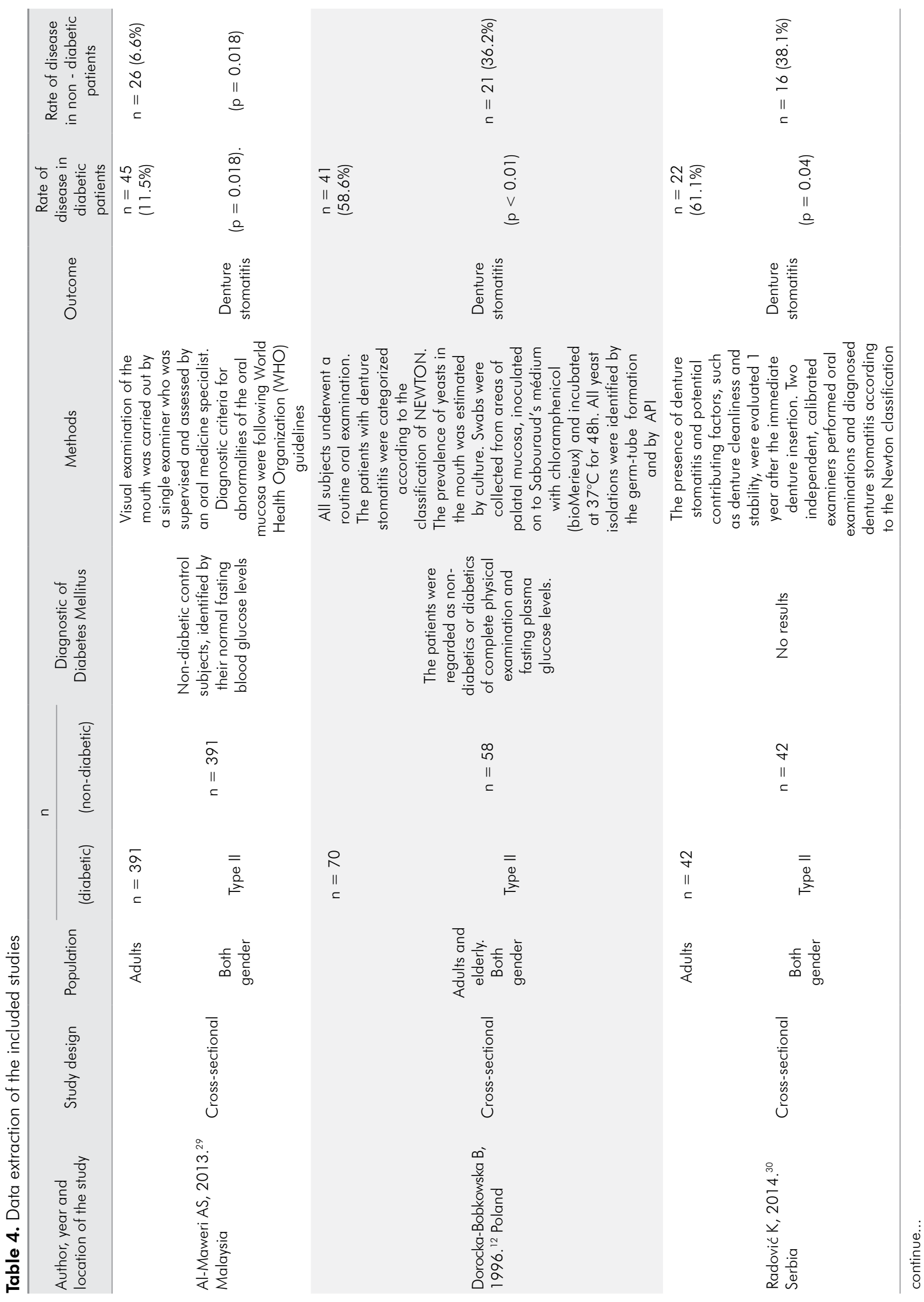




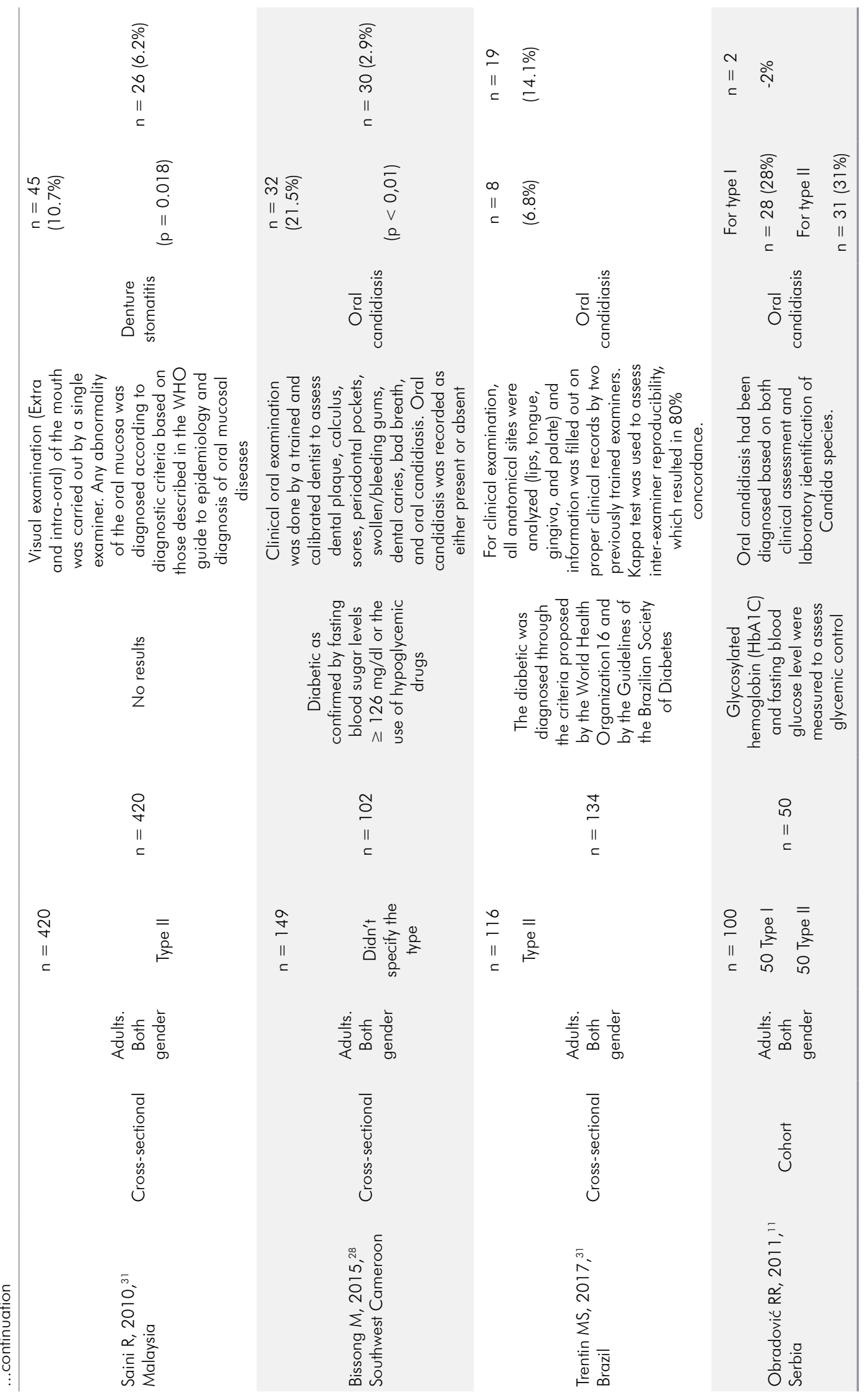


Oral candidiasis and denture stomatitis in diabetic patients: Systematic review and meta-analysis

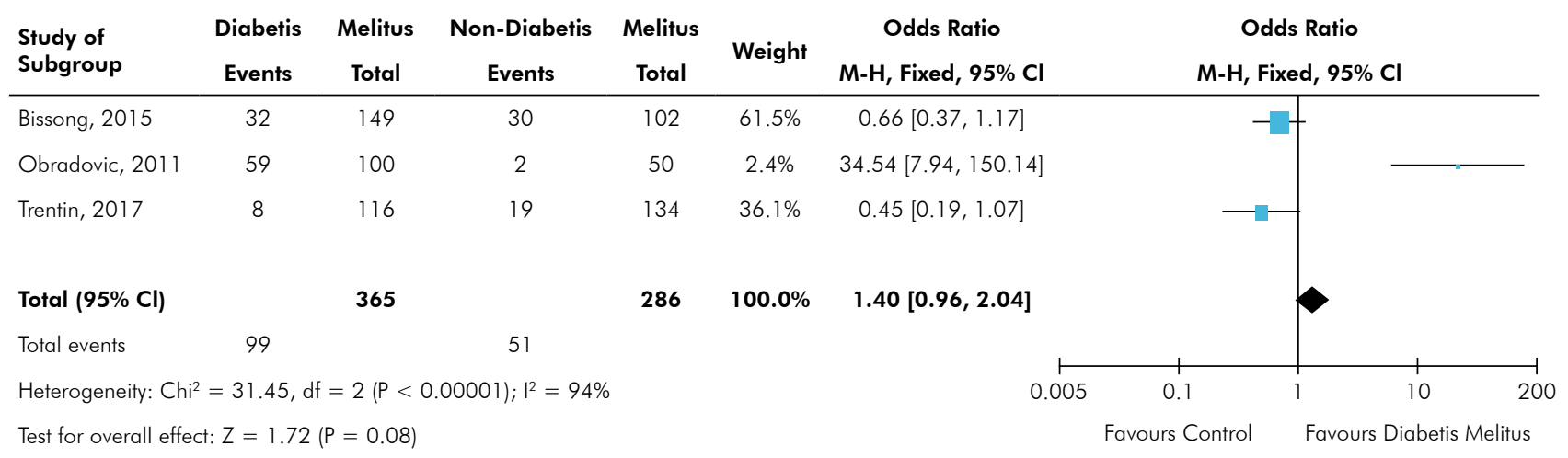

Figure 2. Forest plot of association between oral candidiasis and diabetes mellitus.

\begin{tabular}{|c|c|c|c|c|c|c|c|c|c|c|c|}
\hline Study of Subgroup & $\begin{array}{l}\text { Diabetis } \\
\text { Events }\end{array}$ & $\begin{array}{c}\text { Melitus } \\
\text { Total }\end{array}$ & $\begin{array}{c}\text { Non-Diabetis } \\
\text { Events }\end{array}$ & $\begin{array}{c}\text { Melitus } \\
\text { Total }\end{array}$ & Weight & M-H, Fixed, $95 \% \mathrm{Cl}$ & \multicolumn{5}{|c|}{$\begin{array}{c}\text { Odds Ratio } \\
\mathrm{M-H}, \text { Fixed, } 95 \% \mathrm{Cl}\end{array}$} \\
\hline Al Maweri, 2013 & 45 & 391 & 26 & 391 & $36.3 \%$ & \multicolumn{2}{|l|}{$1.83[1.10,3.02]$} & & - & & \\
\hline $\begin{array}{l}\text { Dorocka-Bobkowska, } \\
1996\end{array}$ & 41 & 70 & 21 & 58 & $15.0 \%$ & $2.49[1.22,5.10]$ & & & & & \\
\hline Rodovic, 2014 & 22 & 42 & 16 & 42 & $12.0 \%$ & $1.79[0.75,4.26]$ & & & & & \\
\hline Saini, 2010 & 45 & 420 & 26 & 420 & $36.6 \%$ & $1.82[1.10,3.01]$ & & & - & & \\
\hline Total $(95 \% \mathrm{Cl})$ & & 923 & & 911 & $100.0 \%$ & $1.92[1.42,2.59]$ & & & & & \\
\hline Total events & 153 & & 89 & & & & & & & & \\
\hline \multicolumn{4}{|c|}{ Heterogeneity: $C h i^{2}=0.62, d f=3(P=0.89) ; I^{2}=0 \%$} & & & 0.1 & 0.2 & 0.5 & 1 & 5 & 10 \\
\hline \multicolumn{4}{|c|}{ Test for overall effect: $Z=4.26(P<0.0001)$} & & & & Favou & Control & Favours Dic & mo & \\
\hline
\end{tabular}

Figure 3. Forest plot of the association between denture stomatitis and diabetes mellitus.

Table 5. Quality of evidence: Association of diabetes mellitus with oral candidiasis and denture stomatitis.

\begin{tabular}{|c|c|c|c|c|c|c|c|c|c|c|}
\hline \multirow{2}{*}{$\begin{array}{l}\text { Participants } \\
\text { (studies) }\end{array}$} & \multicolumn{6}{|c|}{ Certainty assessment } & \multicolumn{4}{|c|}{ Summary of findings } \\
\hline & & & & & & & Study event rates (\%) & & Anticipated $a b$ & olute effects \\
\hline Follow up & $\begin{array}{l}\text { Risk of } \\
\text { bias }\end{array}$ & Inconsistency & Indirectness & Imprecision & $\begin{array}{c}\text { Other } \\
\text { considerations }\end{array}$ & $\begin{array}{l}\text { Overall } \\
\text { certainty of } \\
\text { evidence }\end{array}$ & $\begin{array}{cc}\text { with } & \text { with } \\
\text { non-diabetics } & \text { diabetics }\end{array}$ & $\begin{array}{c}\text { Relative } \\
\text { effect } \\
(95 \% \mathrm{Cl})\end{array}$ & $\begin{array}{c}\text { risk with } \\
\text { non-diabetics }\end{array}$ & $\begin{array}{c}\text { risk } \\
\text { difference } \\
\text { with } \\
\text { diabetics }\end{array}$ \\
\hline
\end{tabular}

\begin{tabular}{|c|c|c|c|c|c|c|c|c|c|c|c|}
\hline \multirow[b]{2}{*}{$\begin{array}{l}651 \\
\text { (3 observational } \\
\text { studies) }\end{array}$} & \multirow[b]{2}{*}{ serious $^{a}$} & \multirow[b]{2}{*}{ very serious ${ }^{b}$} & \multirow[b]{2}{*}{$\begin{array}{c}\text { not } \\
\text { serious }\end{array}$} & \multirow[b]{2}{*}{$\begin{array}{c}\text { very } \\
\text { serious }^{\mathrm{c}, \mathrm{d}}\end{array}$} & \multicolumn{3}{|c|}{ Oral candidiasis } & \multirow[b]{2}{*}{$\begin{array}{l}99 / 365 \\
(27.1 \%)\end{array}$} & \multirow[b]{2}{*}{$\begin{array}{l}\text { OR } 1.40 \\
(0.96 \text { to } \\
2.04)\end{array}$} & \multirow[b]{2}{*}{$\begin{array}{c}178 \text { per } \\
1.000\end{array}$} & \multirow[b]{2}{*}{$\begin{array}{c}55 \text { more } \\
\text { per } 1.000 \\
\text { (from } 6 \\
\text { fewer to } \\
129 \text { more) }\end{array}$} \\
\hline & & & & & none & $\begin{array}{l}\oplus O O O \\
\text { VERY LOW }\end{array}$ & $\begin{array}{l}51 / 286 \\
(17.8 \%)\end{array}$ & & & & \\
\hline \multicolumn{12}{|c|}{ Denture stomatitis } \\
\hline $\begin{array}{l}1834 \\
\text { (4 observational } \\
\text { studies) }\end{array}$ & $\begin{array}{c}\text { not } \\
\text { serious }\end{array}$ & not serious & serious $^{e}$ & $\begin{array}{c}\text { very } \\
\text { serious }^{\mathrm{c}, \mathrm{d}}\end{array}$ & none & $\begin{array}{l}\oplus O O O \\
\text { VERY LOW }\end{array}$ & $\begin{array}{c}89 / 911 \\
(9.8 \%)\end{array}$ & $\begin{array}{c}153 / 923 \\
(16.6 \%)\end{array}$ & $\begin{array}{c}\text { OR } 1.92 \\
(1.42 \text { to } \\
2.59)\end{array}$ & 98 per 1.000 & $\begin{array}{c}74 \text { more } \\
\text { per } 1.000 \\
\text { (from } 36 \\
\text { more to } \\
121 \text { more) }\end{array}$ \\
\hline
\end{tabular}

$\mathrm{Cl}$ : Confidence interval; OR: Odds ratio; ${ }^{*}$ Other considerations include publication bias, magnitude of effect, plausible confounding factors, and analysis of spurious effects. Explanations: $a$. All included studies presented some type of risk of bias; b. There was wide variation in the effect estimates across studies, a little overlap of confidence intervals associated with the effect estimates, and high and significant heterogeneity; c. Upper and lower confidence interval limits were greater than $25 \%$ of OR; d. Total number of events was less than 300 ; e. All studies assessed particular versions of the exposition (Diabetes type II). 
Besides the requirement to pair participants, the inclusion and exclusion of certain criteria should be carefully established. There was a lack of clarity in some studies in this systematic review ${ }^{11,28,30,31}$ on these parameters. For instance, the authors stated that patients were exclusively diabetic with no other diseases; however, they did not state whether smokers and alcoholics were excluded. Because these conditions are not characterized as systemic diseases, patients with these conditions might have been included, generating a serious risk of bias.

This systematic review also evaluated the quality control of the studies, with respect to validity, reproducibility, and blindness. Unexpectedly all studies had problems with respect to these components. Regarding validity, which is using a validated diagnostic method for evaluating the presence of oral candidiasis and denture stomatitis, two studies ${ }^{29.31}$ followed the recommendations described by the World Health Organization (WHO) guideline, while two others ${ }^{12,30}$ followed the Newton Classification of Oral Candidiasis. However, three studies ${ }^{11,28,32}$ did not follow any guideline; consequently, these data might be underestimated or overestimated. To provide reliable data and ensure the reproducibility, epidemiological studies must follow validated data collection parameters.

Besides following a validated protocol, reproducibility is also related to examiner calibration for the method to be used. Only one study ${ }^{32}$ had two independent calibrated examiners, and provided the Kappa values. Some studies $29,30,31$ had specialists as examiners, while three ${ }^{11,12,28}$ did not use calibrated examiners or specialist opinions. Diagnosis by a calibrated examiner ensures that oral candidiasis and denture stomatitis are assessed properly, confirming reliability.

The blindness of the studies might represent another major risk of bias. No study blinded the examiners to evaluate the groups (diabetics or healthy) or to perform the statistical analysis. Blindness is essential to guarantee that the examiner did not influence or induce the results. Consequently, all studies were classified as having major problems. Thus, future studies should consider blinding evaluators.

After evaluating the methodological quality of the articles, two meta-analyses were performed.
The first meta-analysis showed that diabetic patients have a similar chance of developing oral candidiasis compared to non-diabetic patients. Thus diabetes mellitus could not be directly related to oral candidiasis. However, this result should be analyzed cautiously, due to methodological problems found in studies. Oral candidiasis has known predisposing factors in individuals who are compromised by systemic conditions (e.g., immunosuppression) or who have had tissue or organ transplants, developing malignancies, or immune diseases, such as HIV patients. ${ }^{35}$ On the other hand, denture stomatitis is related to poor denture fit, greater age of the denture user, greater age of dentures, and poor denture hygiene. ${ }^{36}$ Thus, we hypothesized that the use of dentures could influence the presence of the disease.

In the second meta-analysis evaluating denture stomatitis as an outcome, diabetic patients had a higher chance of having denture stomatitis compared to healthy patients. Thus, removable prostheses are a risk factor for the colonization and development of denture stomatitis. Removable prostheses promote a favorable microenvironment for the growth of Candida albicans. The oral mucosal-prosthesis interface has low levels of oxygen and $\mathrm{pH},{ }^{37}$ which are associated with poor hygiene ${ }^{38}$ and reduced salivary flow. These factors likely favor the colonization of $C$. albicans, and subsequent development of denture stomatitis. However, the results of this second metaanalysis must be analyzed carefully, because the studies had methodological problems that might have compromised the results. Thus, future studies should consider evaluating prosthetic conditions and timing of use, since the degradation of the dentures over time could be a factor that favors Candida colonization.

Of importance, several components might contribute to the high heterogeneity among studies. Examples include the age of denture wearers, female individuals, smoking habits, and compromised immune system. Appropriate assessment is essential for diagnosing denture stomatitis, and should be based on methods detecting or grading Candida in a scoring system. Out of the scoring systems, Newton Classification of Oral Candidiasis and the World 
Health Organization (WHO) guideline were the most frequently cited methods in the retrieved papers. However, most publications did not mention any method for evaluating this phenomenon, other than visually, which might have influenced the results of these studies. ${ }^{39}$

Despite this systematic review covering a large number of articles in the search process, publication bias might also exist. It is easier to find studies with positive results indexed within search databases, but which might not include all studies about this topic. To minimize publication bias, this systematic review also included searching the gray literature and hand-searching. Another limitation of this systematic review was the power to generate strong evidence, due to the methodological problems found in the studies. Therefore, the certainty of the evidence (GRADE) was considered very low. Thus, more primary studies should be designed to generate sufficient evidence on the prevalence of oral candidiasis and denture stomatitis among diabetic individuals.

Although the quality of the evidence was very low, the results of this systematic review and meta-analysis showed that there was a higher prevalence of denture stomatitis when patients with diabetes mellitus were compared with healthy patients. Of importance, undiagnosed and untreated diabetic individuals might have a higher risk of developing Candida infection. ${ }^{39}$ Thus, our study also highlights dentists should investigate the cause of candidiasis among their patients and verify if they are diabetics patients. Such information could help with the early diagnosis of diabetes, leading to better treatment strategies.
The results obtained by the current study reinforced the importance of stressing that diabetic patients should lead a healthy lifestyle with good oral and dental prosthesis hygiene. These initiatives help to prevent Candida infection, promoting a better quality of life for these patients. Considering that diabetes is a highly prevalent disease, and is a public health problem globally, medical specialists responsible for diabetic patient care should inform them to have dentist follow-ups to prevent oral candidiasis and denture stomatitis. In addition, treatment centers for diabetic patients with a multi-professional approach should include dentists. This inclusion could facilitate the early diagnosis of oral candidiasis and, consequently, better treatment and prevention initiatives.

\section{Conclusions}

Diabetic patients have a similar chance of developing oral candidiasis compared to nondiabetic patients. Moreover, diabetic individuals have a higher chance of presenting with denture stomatitis compared to healthy patients. Yet, the certainty of evidence was very low. Consequently, these data should be interpreted with caution, due to the methodological problems that might influence the results. In conclusion, future studies should consider enhancing the quality of the methods used.

\section{Acknowledgments}

The authors thank the Coordenação de Aperfeiçoamento de Pessoal de Nível Superior Brazil (CAPES) for providing scholarships to the first, second, third, and fourth authors. Finance Code 001.

\section{References}

1. Bakhti M, Böttcher A, Lickert H. Modelling the endocrine pancreas in health and disease. Nat Rev Endocrinol. 2019 Mar;15(3):155-71. https://doi.org/10.1038/s41574-018-0132-z

2. Mathers CD, Loncar D. Projections of global mortality and burden of disease from 2002 to 2030. PLoS Med. 2006 Nov;3(11):e442. https://doi.org/10.1371/journal.pmed.0030442

3. Tao Z, Shi A, Zhao J. Epidemiological perspectives of diabetes. Cell Biochem Biophys. 2015 Sep;73(1):181-5. https://doi.org/10.1007/s12013-015-0598-4

4. Bastos AS, Leite AR, Spin-Neto R, Nassar PO, Massucato EM, Orrico SR. Diabetes mellitus and oral mucosa alterations: prevalence and risk factors. Diabetes Res Clin Pract. 2011 Apr;92(1):100-5. https://doi.org/10.1016/j.diabres.2011.01.011 
5. Knop MR, Geng TT, Gorny AW, Ding R, Li C, Ley SH, et al. Birth weight and risk of type 2 diabetes mellitus, cardiovascular disease, and hypertension in adults: a meta-analysis of 7646267 participants from 135 studies. J Am Heart Assoc. 2018 Dec;7(23):e008870. https://doi.org/10.1161/JAHA.118.008870

6. Gao B, Wu S, Wang J, Yang C, Chen S, Hou J, et al. Clinical features and long-term outcomes of diabetic kidney disease: a prospective cohort study from China. J Diabetes Complications. 2019 Jan;33(1):39-45. https://doi.org/10.1016/i.jdiacomp.2018.09.019

7. Kim SW, Kang GW. Diabetes mellitus as a risk factor for glaucoma outcome in Korea. Acta Ophthalmol. 2017 Nov;95(7):e662-4. https://doi.org/10.1111/aos.13345

8. Lima AL, Illing T, Schliemann S, Elsner P. Cutaneous manifestations of diabetes mellitus: a review. Am J Clin Dermatol. 2017 Aug;18(4):541-53. https://doi.org/10.1007/s40257-017-0275-z

9. Zhou X, Zhang W, Liu X, Zhang W, Li Y. Interrelationship between diabetes and periodontitis: role of hyperlipidemia. Arch Oral Biol. 2015 Apr;60(4):667-74. https://doi.org/10.1016/i.archoralbio.2014.11.008

10. Jhugroo C, Divakar DD, Jhugroo P, Al-Amri SA, Alahmari AD, Vijaykumar S, et al. Characterization of oral mucosa lesions and prevalence of yeasts in diabetic patients: a comparative study. Microb Pathog. 2019 Jan;126:363-7. https://doi.org/10.1016/j.micpath.2018.11.028

11. Obradović RR, Kesić LG, Pejčić AA, Petrović MS, Živković ND, Živković DM. Diabetes mellitus and oral candidiasis. Acta Stomatol Naissi. 2011;27(63):1025-34. https://doi.org/10.5937/asn11630250

12. Dorocka-Bobkowska B, Budtz-Jörgensen E, Włoch S. Non-insulin-dependent diabetes mellitus as a risk factor for denture stomatitis. J Oral Pathol Med. 1996 Sep;25(8):411-5. https://doi.org/10.1111/j.1600-0714.1996.tb00288.x

13. Wikner C, Gigante B, Hellénius ML, de Faire U, Leander K. The risk of type 2 diabetes in men is synergistically affected by parental history of diabetes and overweight. PLoS One. 2013 Apr;8(4):e61763. https://doi.org/10.1371/journal.pone.0061763

14. Peleg AY, Hogan DA, Mylonakis E. Medically important bacterial-fungal interactions. Nat Rev Microbiol. 2010 May;8(5):340-9. https://doi.org/10.1038/nrmicro2313

15. Buu LM, Chen YC. Impact of glucose levels on expression of hypha-associated secreted aspartyl proteinases in Candida albicans. J Biomed Sci. 2014 Mar;21(1):22. https://doi.org/10.1186/1423-0127-21-22

16. Contaldo M, Romano A, Mascitti M, Fiori F, Della Vella F, Serpico R, et al. Association between denture stomatitis, Candida species and diabetic status. J Biol Regul Homeost Agents. 2019 May-Jun;33(3 Suppl. 1):35-41.

17. Kadir T, Pisiriciler R, Akyüz S, Yarat A, Emekli N, Ipbüker A. Mycological and cytological examination of oral candidal carriage in diabetic patients and non-diabetic control subjects: thorough analysis of local aetiologic and systemic factors. J Oral Rehabil.

2002 May;29(5):452-7. https://doi.org/10.1046/j.1365-2842.2002.00837.x

18. Daniluk T, Tokajuk G, Stokowska W, Fiedoruk K, Sciepuk M, Zaremba ML, et al. Occurrence rate of oral Candida albicans in denture wearer patients. Adv Med Sci. 2006;51 Suppl 1:77-80.

19. Rajakumari ML, Saravana Kumari P. Prevalence of Candida species in the buccal cavity of diabetic and non-diabetic individuals in and around Pondicherry. J Mycol Med. 2016 Dec;26(4):359-67. https://doi.org/10.1016/j.mycmed.2016.08.002

20. Mohammadi F, Javaheri MR, Nekoeian S, Dehghan P. Identification of Candida species in the oral cavity of diabetic patients. Curr Med Mycol. 2016 Jun;2(2):1-7. https://doi.org/10.18869/acadpub.cmm.2.2.4

21. Moher D, Shamseer L, Clarke M, Ghersi D, Liberati A, Petticrew M, et al. Preferred reporting items for systematic review and metaanalysis protocols (PRISMA-P) 2015 statement. Syst Rev. 2015 Jan;4(1):1. https://doi.org/10.1186/2046-4053-4-1

22. Maia LC, Antonio AG. Systematic reviews in dental research: a guideline. J Clin Pediatr Dent. 2012;37(2):117-24. https://doi.org/10.17796/jcpd.37.2.h606137vj3826v61

23. Fowkes FG, Fulton PM. Critical appraisal of published research: introductory guidelines. BMJ. 1991 May;302(6785):1136-40. https://doi.org/10.1136/bmi.302.6785.1136

24. Penoni DC, Fidalgo TK, Torres SR, Varela VM, Masterson D, Leão AT, et al. Bone density and clinical periodontal attachment in postmenopausal women: a systematic review and meta-analysis. J Dent Res. 2017 Mar;96(3):261-9. https://doi.org/10.1177/0022034516682017

25. Fernandes LM, Cordeiro Neto J, Lima TF, Magno MB, Santiago BM, Cavalcanti YW, et al. The use of mouthguards and prevalence of dento-alveolar trauma among athletes: a systematic review and meta-analysis. Dent Traumatol. 2019 Feb;35(1):54-72. https://doi.org/10.1111/edt.12441

26. Borenstein M, Hedges LV, Higgins JP, Rothstein HR. Introduction to meta-analysis. New York: John Wiley \& Sons; 2009.

27. Atkins D, Eccles M, Flottorp S, Guyatt GH, Henry D, Hill S, et al. Systems for grading the quality of evidence and the strength of recommendations I: critical appraisal of existing approaches. BMC Health Serv Res. 2004 Dec;4(1):38. https://doi.org/10.1186/1472-6963-4-38

28. Bissong M, Azodo CC, Agbor MA, Nkuo-Akenji T, Fon PN. Oral health status of diabetes mellitus patients in Southwest Cameroon. Odontostomatol Trop. 2015 Jun;38(150):49-57. 
Oral candidiasis and denture stomatitis in diabetic patients: Systematic review and meta-analysis

29. Al-Maweri SA, Ismail NM, Ismail AR, Al-Ghashm A. Prevalence of oral mucosal lesions in patients with type 2 diabetes attending Hospital Universiti Sains Malaysia. Malays J Med Sci. 2013 Jul;20(4):39-46.

30. Radović K, llić J, Roganović J, Stojić D, Brković B, Pudar G. Denture stomatitis and salivary vascular endothelial growth factor in immediate complete denture wearers with type 2 diabetes. J Prosthet Dent. 2014 May; 111(5):373-9. https://doi.org/10.1016/i.prosdent.2013.07.019

31. Saini R, Al-Maweri SA, Saini D, Ismail NM, Ismail AR. Oral mucosal lesions in non oral habit diabetic patients and association of diabetes mellitus with oral precancerous lesions. Diabetes Res Clin Pract. 2010 Sep;89(3):320-6. https://doi.org/10.1016/i.diabres.2010.04.016

32. Trentin MS, Verardi G, Ferreira MC, Carli JP, Silva SO, Lima IF, et al. Most frequent oral lesions in patients with type 2 diabetes mellitus. J Contemp Dent Pract. 2017 Feb;18(2):107-11. https://doi.org/10.5005/ip-journals-10024-1999

33. Guggenheimer J, Moore PA, Rossie K, Myers D, Mongelluzzo MB, Block HM, et al. Insulin-dependent diabetes mellitus and oral soft tissue pathologies. I. Prevalence and characteristics of non-candidal lesions. Oral Surg Oral Med Oral Pathol Oral Radiol Endod. 2000 May;89(5):563-9. https://doi.org/10.1067/moe.2000.104476

34. Collin HL, Niskanen L, Uusitupa M, Töyry J, Collin P, Koivisto AM, et al. Oral symptoms and signs in elderly patients with type 2 diabetes mellitus: a focus on diabetic neuropathy. Oral Surg Oral Med Oral Pathol Oral Radiol Endod. 2000 Sep;90(3):299-305. https://doi.org/10.1067/moe.2000.107536

35. Millsop JW, Fazel N. Oral candidiasis. Clin Dermatol. 2016 Jul-Aug;34(4):487-94. https://doi.org/10.1016/j.clindermatol.2016.02.022

36. Gendreau L, Loewy ZG. Epidemiology and etiology of denture stomatitis. J Prosthodont. 2011 Jun;20(4):251-60. https://doi.org/10.1111/j.1532-849X.2011.00698.x

37. Martori E, Ayuso-Montero R, Willaert E, Viñas M, Peraire M, Martinez-Gomis J. Status of removable dentures and relationship with oral candida-associated factors in a geriatric population in Catalonia. J Prosthodont. 2017 Jul;26(5):370-5. https://doi.org/10.1111/jopr.12551

38. Serefko AD, Poleszak EJ, Malm A. Candida albicans denture biofilm and its clinical significance. Pol J Microbiol. 2012 Sep;61(3):161-7. https://doi.org/10.33073/pim-2012-021

39. Zheng Y, Ley SH, Hu FB. Global aetiology and epidemiology of type 2 diabetes mellitus and its complications. Nat Rev Endocrinol. 2018 Feb;14(2):88-98. https://doi.org/10.1038/nrendo.2017.151 